Document downloaded from:

http://hdl.handle.net/10251/105469

This paper must be cited as:

Gregori Verdú, S.; Tur Valiente, M.; Nadal, E.; Fuenmayor Fernández, F. (2017). An approach to geometric optimisation of railway catenaries. Vehicle System Dynamics. 1-25. doi:10.1080/00423114.2017.1407434

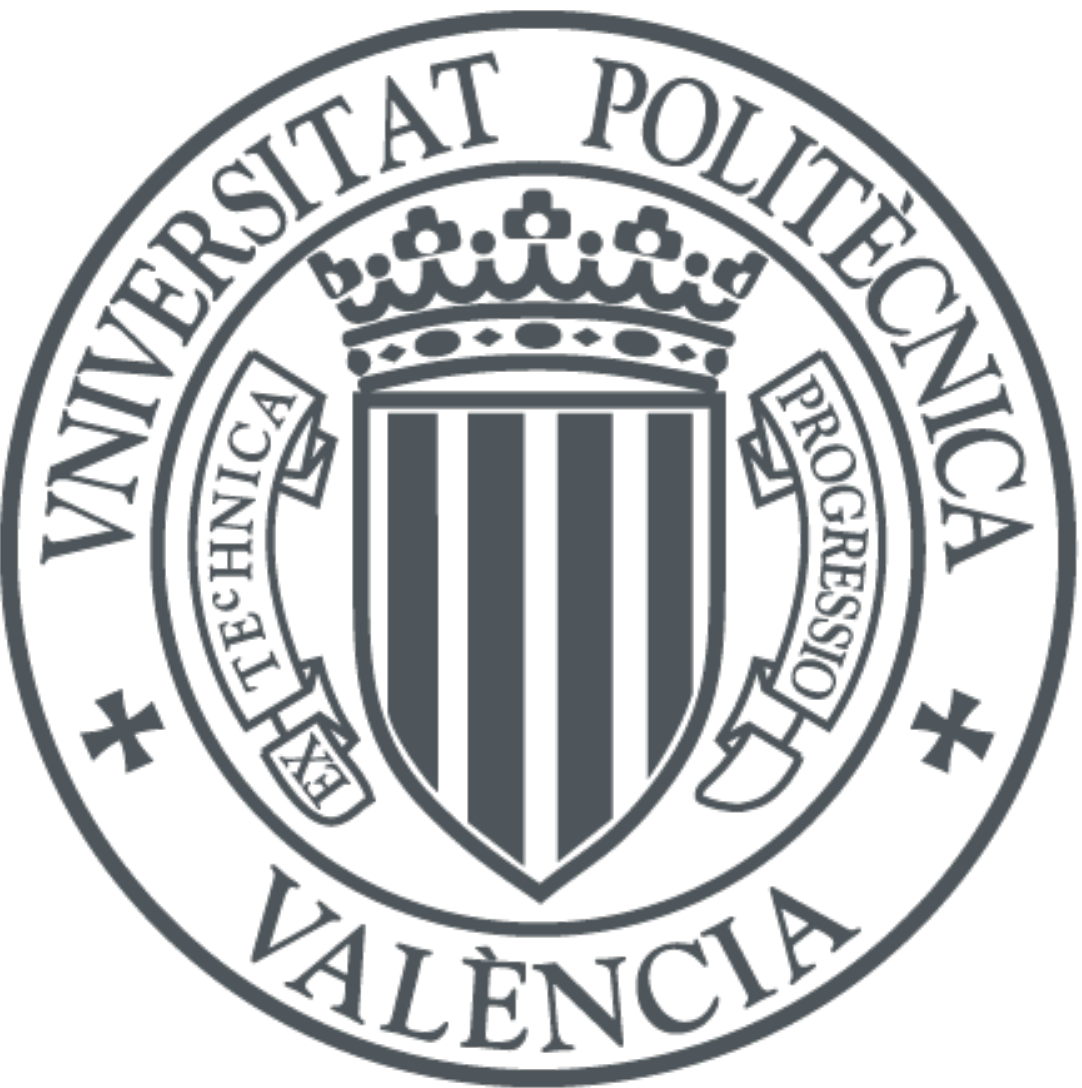

The final publication is available at

https://doi.org/10.1080/00423114.2017.1407434

Copyright Taylor \& Francis

Additional Information 


\title{
An approach to geometric optimisation of railway catenaries
}

\author{
S. Gregori ${ }^{a}$, M. Tur ${ }^{a}$, E. Nadal $^{a}$, F.J. Fuenmayor ${ }^{a}$, \\ ${ }^{a}$ Centro de Investigación en Ingeniería Mecánica, \\ Departamento de Ingeniería Mecánica y de Materiales, Universitat Politècnica de València, \\ Camino de Vera s/n, 46022 Valencia, Spain
}

April, 2017

\begin{abstract}
The quality of current collection becomes a limiting factor when the aim is to increase the speed of the present railway systems. In this work an attempt is made to improve current collection quality optimising catenary geometry by means of a Genetic Algorithm. As dropper lengths and dropper spacing are thought to be highly influential parameters they were chosen as the optimisation variables. The results obtained show that a Genetic Algorithm can be used to optimise catenary geometry to improve current collection quality measured in terms of the standard deviation of the contact force. Furthermore, it is highlighted that apart from the usual pre-sag, other geometric parameters should also be taken into account when designing railway catenaries.
\end{abstract}

\section{Introduction}

The overhead equipment, commonly named the catenary, is the system in charge of providing the energy supply to the electric vehicle by means of a sliding contact with the 
pantograph on top of the locomotive. The interaction force between the pantograph and the catenary contact wire determines the quality of the supply. High contact forces can cause excessive wear on the sliding surfaces, while too weak forces may lead to contact losses and sparking, which apart from the damage it can cause, interrupts the energy supply. As the maximum speed of commercial railways is mainly limited by the pantographcatenary interaction $[1,2]$, an appropriate design is crucial for the correct behaviour of such a system.

This explains why in recent years a lot of effort has been put into developing accurate models capable of simulating the dynamic pantograph-catenary interaction. Among the vast diversity of studies found in the literature, [3-13] deserve special mention as they participated in a benchmark [14], and give a good insight into the present state of the art.

Many parameters influence current collection quality, the pantograph being one of the most important, which is why it has been extensively covered in the literature. Its influence was studied in [15] and some optimisations are given concerning the parameters which define the model in [16-19]. As regards the catenary itself, the effect of different parameters such as span length [20] or wire tensions have also been widely studied, along with the amount of initial sag (pre-sag) given to the contact wire, which also seems to be a key factor in current collection quality [21-23]. However, certain discrepancies can be found in these references concerning the benefits of pre-sag on current collection quality. Another aspect which has received slight attention in the literature is dropper spacing, which can also strongly affect the interaction force.

The present study, up tp the authors knowledge, is the first attempt in finding the optimal catenary geometry by exploring other alternatives, such as the contact wire height profile and dropper spacing, and analyses two catenary topologies: with and without a stitch wire. The catenary system is modelled by the Finite Element Method (FEM) and a lumped-parameters model is used to represent the pantograph. The optimisation problems are solved by means of a genetic algorithm with some operational restrictions.

The paper is organised as follows: after this brief introduction, all the mathematical models used to describe the whole system are explained in Section 2. The initial configuration problem of the catenary is treated in Section 3, while Section 4 is devoted to explaining the dynamic interaction problem, which is solved efficiently. These three sections are 
included for the sake of completeness since they provide a summary of the models presented in [9], the shape-finding problem solved in [24] and the time integration procedure proposed in [25], respectively. The optimisation problem is set in Section 5, together with the Genetic Algorithm used to solve it. The results obtained from these optimisations are given in Section 6 for both catenary topologies. Finally, the main conclusions drawn from this work are offered in Section 7.

\section{Catenary, pantograph and interaction models}

The FE technique is the most widely used to model high-speed railway catenaries as can be seen from the review [26] and references in [14]. The catenary is mainly composed of a messenger wire, a contact wire, registration arms, droppers and some typologies also have stitch wires as can be seen in Fig. 1. In this work, the messenger and the contact wires are modelled by beam elements based on the Absolute Nodal Coordinate Formulation (ANCF), which account for axial and bending deformations, and they are identified as 'cable elements' throughout this paper. This type of element was first proposed by Shabana [27] and adapted for thin beams and cables in [28]. Unlike other beam formulations, ANCF elements use absolute positions and their gradients as degrees of freedom instead of rotations [24]. Bar elements are used to model droppers, registration arms and stitch wires, since they only transmit axial forces.

The reference and deformed configurations for a cable element are schematically represented in Fig. 2. In order to guarantee $C^{1}$ continuity, standard Hermite elements are used in which the length of the undeformed element $l_{r e f}^{e}$ is present.

The degrees of freedom of bar elements are only composed of the absolute positions of the two nodes of the element, so that a linear interpolation is enough to ensure the required $C^{0}$ continuity, since bending deformations are not taken into account.

A wide variety of solutions can be found to model pantographs [19]. For its simplicity a linear lumped-parameters model is used in this work that only introduces three vertical degrees of freedom, as shown in the scheme depicted in Fig. 3a. $F_{p}$ represents the force exerted by the uplift mechanism, which acts on the lower mass of the pantograph. 


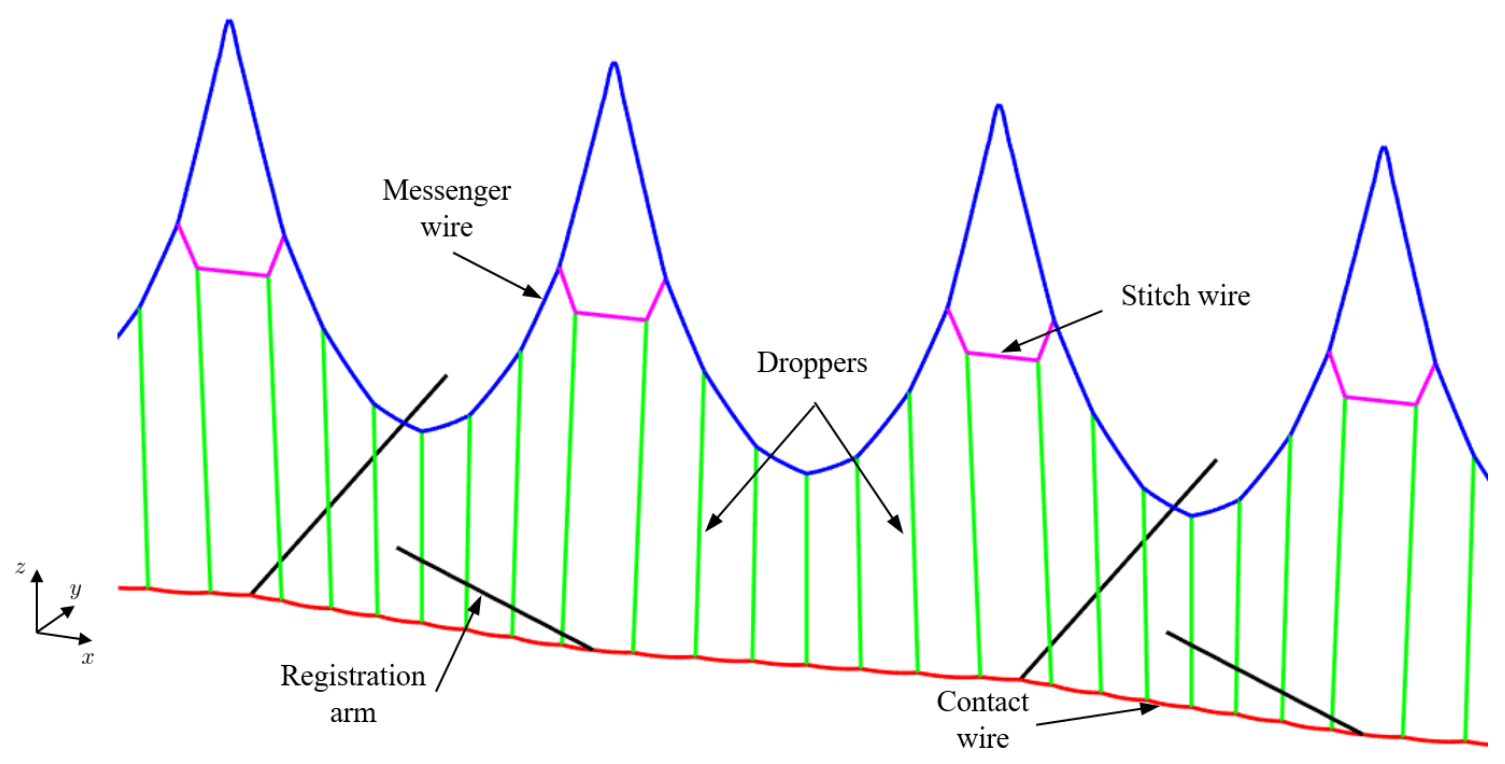

Figure 1: Finite element catenary model.
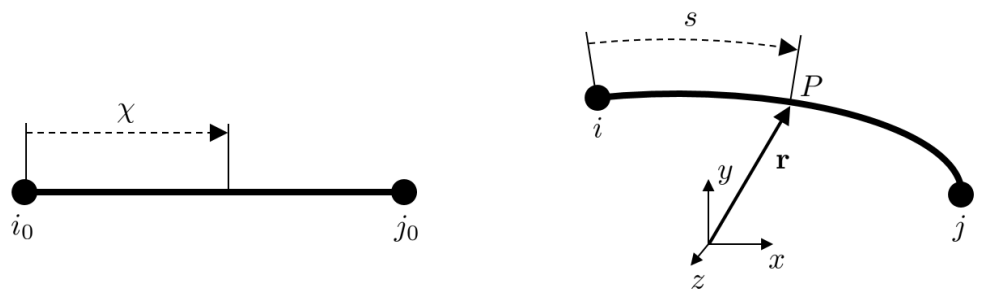

Figure 2: Reference and deformed configurations of the ANCF element.

A penalty method is considered to model the pantograph-catenary interaction. A scheme of this interaction is shown in Fig. 3b. In this model, a spring element with high stiffness $k_{h}$ relates the vertical degrees of freedom of the contact cable element with the pantograph upper mass. The interaction force is obtained by multiplying $k_{h}$ by the interpenetration, i.e.:

$$
f_{\text {inter }}=\left\{\begin{array}{ccc}
k_{h}\left(z_{1}-z_{c w}\right) & \text { if } & z_{1}-z_{c w}>0 \\
0 & \text { if } & z_{1}-z_{c w} \leq 0
\end{array}\right.
$$

where $z_{1}$ and $z_{c w}$ are the absolute vertical position of the upper mass of the pantograph and the contact point on the contact wire, respectively. 


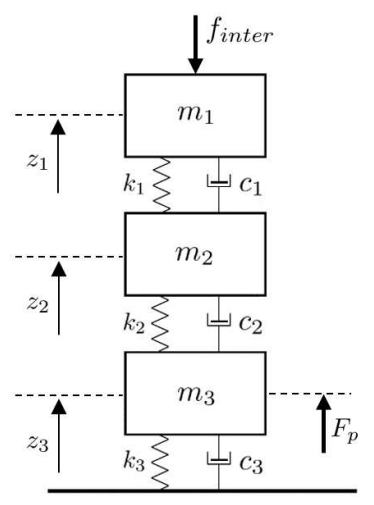

(a)

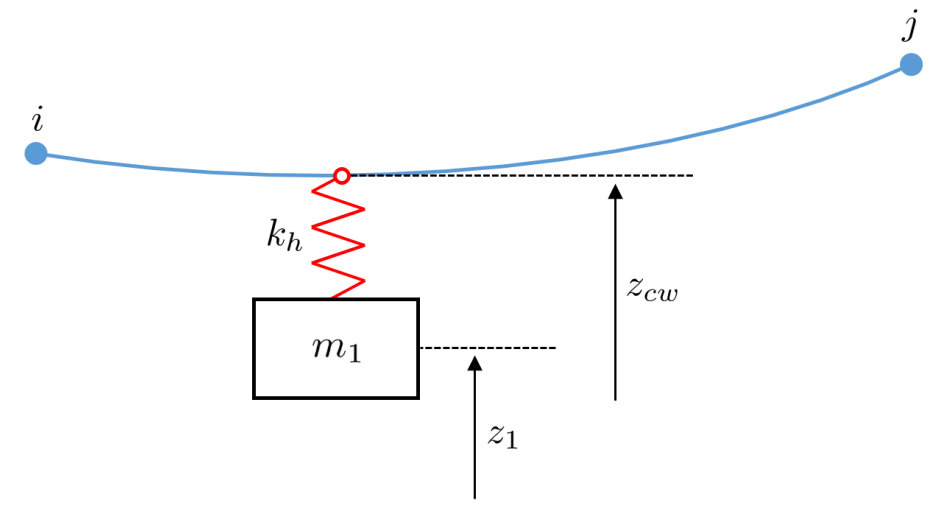

(b)

Figure 3: (a) Pantograph and (b) interaction model schemes.

\section{Initial configuration problem}

The initial configuration or 'shape-finding' problem, consists of finding the position of each node along with the non-deformed length of each element in the mesh which fulfils both the static equilibrium equations and the constraints imposed by the stringing process. Due to the large displacements undergone by the cabling this is a non-linear problem. Despite the approach and the solution procedure are thoroughly explained in [24], some insights are given here.

Following the previous reference, the static equilibrium problem is defined by means of the non-linear equation:

$$
\mathbf{F}_{\text {int }}\left(\mathbf{q}, \mathbf{l}_{\text {ref }}^{e}\right)+\mathbf{F}_{g}\left(\mathbf{l}_{\text {ref }}^{e}\right)=0
$$

along with the appropriate essential boundary conditions. The internal forces $\mathbf{F}_{\text {int }}$ depend on the nodal coordinates $\mathbf{q}$ as well as the reference lengths of the elements $\mathbf{1}_{r e f}^{e}$, while the gravitational forces $\mathbf{F}_{g}$ only depend on the latter. For a given element's length, $\mathbf{l}_{\text {ref }}^{e}$, Eq. (2) can be solved by using for example the Newton-Raphson method, in order to obtain the static equilibrium position of the system.

However, the final static equilibrium position of the cabling must fulfil certain constraints apart from the force equilibrium. Certain elements such as those modelling the messenger wire, contact wire and stitch wire, are pre-stressed with a tension of value $T$. In a given 
element $e$, this constraint can be described as:

$$
c_{I}\left(\mathbf{q}, l_{\text {ref }}\right)=\left(f_{\text {int }_{x}}^{e}\right)^{2}+\left(f_{\text {inty }_{y}}^{e}\right)^{2}+\left(f_{\text {int } t_{z}}^{e}\right)^{2}-T^{2}=0
$$

where $\left(f_{\text {int }_{j}}^{e}\right)$ is the $j$ component of the internal nodal force vector. Other constraints such as the contact wire height, and the horizontal position of droppers, stitch wires, registration arms and mast supports, are imposed by means of the constraint equation:

$$
c_{I I}(\mathbf{q})=q_{i}-P=0
$$

where $q_{i}$ for $i=x, y z$ is the nodal coordinate, which is enforced to have a value of $P$.

Putting equilibrium equations (2) and constraints $\mathbf{c}\left(\mathbf{q}, \mathbf{l}_{r e f}^{e}\right)$ together results in the nonlinear system of algebraic equations:

$$
\left.\begin{array}{l}
\mathbf{F}\left(\mathbf{q}, \mathbf{l}_{r e f}^{e}\right)=0 \\
\mathbf{c}\left(\mathbf{q}, \mathbf{l}_{r e f}^{e}\right)=0
\end{array}\right\}
$$

which can be solved by the Newton-Raphson method, obtaining the nodal absolute positions $\mathbf{q}$ and the initial length of each element $l_{r e f}^{e}$, which fulfil the restrictions imposed for the catenary stringing.

\section{Dynamic interaction problem}

The pantograph-catenary dynamic interaction is governed by small displacements, therefore the linear system of differential equations:

$$
\mathbf{M u}+\mathbf{C} \dot{\mathbf{u}}+\mathbf{K u}=\mathbf{F}
$$

is suitable for modelling the whole behaviour of the system [25]. The stiffness matrix $\mathbf{K}$ is obtained from linearisation of dynamic equation at the static equilibrium position resulting from solving Eq (5). M, C, are the mass and damping matrices of the whole system. All these three matrices contain both the pantograph and the catenary contributions. $\mathbf{F}$ is the vector of applied external forces and $\mathbf{u}$ denotes the displacements of the pantograph-catenary with respect to its static equilibrium configuration. A proportional 
Rayleigh damping model is considered in Eq. (6). This ordinary differential equation can be solved by using any time integration scheme such as the commonly used Newmark method. In order to obtain the displacements of the actual time step $t$, given the solution in the previous time step, $\mathbf{u}^{t-1}$, the algebraic system of equations:

$$
\hat{\mathbf{K}} \mathbf{u}^{t}=\mathbf{F}_{k n}\left(\mathbf{u}^{t-1}\right)+\mathbf{F}_{d r}^{t}\left(\mathbf{u}^{t}\right)+\mathbf{F}_{\text {inter }}^{t}\left(\mathbf{u}^{t}\right)
$$

must be solved. Matrix $\hat{\mathbf{K}}$ is obtained by applying the Newmark time discretization to Eq. (6), (see [25]). $\mathbf{F}_{k n}$ is the vector of known forces, which depends on information of the previous time step, $\mathbf{F}_{d r}$ is the force vector necessary to compensate the slackened droppers and $\mathbf{F}_{\text {inter }}$ accounts for the interaction force terms. Note that Eq. (7) is a non-linear system since the following non-linearities are considered: i) dropper slackening, which means that droppers only work in tension and, ii) loss of contact between the pantograph and the contact wire, leading to a null interaction force.

In order to speed up the calculations, the approach proposed in [25] is adopted. This method is based on two fundamental ideas. The first idea was originally proposed in [29] and successfully used in [10]. It consists of moving the correction forces of the slackened droppers, $\mathbf{F}_{d r}$, and the terms involving the interaction force, $\mathbf{F}_{\text {inter }}$, to the right hand side of Eq. (7). In this way, the matrix $\hat{\mathbf{K}}$ does not change in time and can be pre-computed and factorised only once in the algorithm.

The second idea of the method relies on the superposition principle. The displacements $\mathbf{u}^{t}$ can be computed as the sum of those displacements produced by the three terms present on the right hand side of Eq. (7), i.e.:

$$
\mathbf{u}^{t}=\mathbf{u}_{k n}+\mathbf{u}_{d r}+\mathbf{u}_{\text {inter }}
$$

To obtain the so-called known term, $\mathbf{u}_{k n}$ (dependent from information of the previous time step), it is necessary to solve the global size system:

$$
\hat{\mathbf{K}} \mathbf{u}_{k n}=\mathbf{F}_{k n}
$$


However, the other two contributions to the total displacement can be written as:

$$
\begin{aligned}
\mathbf{u}_{d r} & =\mathbf{u}_{d r}^{*} f_{d r} \\
\mathbf{u}_{\text {inter }} & =\mathbf{u}_{\text {inter }}^{*} f_{\text {inter }}
\end{aligned}
$$

where $\mathbf{u}_{d r}^{*}$ and $\mathbf{u}_{i n t e r}^{*}$ are the displacements produced by unitary forces applied at any dropper ends and at the interaction point, respectively. Both terms can also be precomputed, leading to a new problem whose unknowns are the values of the slackened droppers' correction forces $f_{d r}$, and the interaction force $f_{\text {inter }}$.

To sum up, the unilateral constraints of the system are iteratively dealt with in a system much reduced in size. This makes the approach highly efficient, requiring a low computational cost to simulate the pantograph-catenary dynamic interaction.

\section{Optimisation problem}

As pointed out in the introduction, the main goal of this paper is to seek the best catenary geometry in terms of current collection quality. Among other parameters, the quotient between standard deviation and mean interaction force $\sigma\left(f_{\text {inter }}\right) / \bar{f}_{\text {inter }}$ is thought to be a representative statistical parameter to characterize the quality of the interaction [17]. The standard [30] sets this parameter below 0.3 , which guarantees less than a $1 \%$ probability of contact loss. This standard also establishes that the interaction force must be low-pass filtered at $20 \mathrm{~Hz}$ and the maximum mean contact force applied to the contact wire must fulfil the relationship:

$$
\bar{f}_{\text {inter }}<0.0097 v^{2}+70
$$

for an alternating current catenary, where $200<v<320 \mathrm{~km} / \mathrm{h}$. The interaction force varies in time and depends on many factors such as train speed, catenary geometry, material properties and so on. In this work, the train speed, the mean contact force, the pantograph model and the wire tensions remain constant, while the geometrical parameters related to droppers (length or spacing) are considered as the optimisation variables for minimizing $\sigma\left(f_{\text {inter }}\right)$.

Generally, denoting as $\mathbf{p}$ the set of parameters with respect to which it is desired to 
optimise the catenary, the optimisation problem reads:

$$
\begin{aligned}
& \min _{\mathbf{p}} \sigma\left(f_{\text {inter }}^{t}(\mathbf{p})\right) \\
& \text { s.t. } \\
& p_{i}^{\text {min }} \leq p_{i} \leq p_{i}^{\max } \quad i=1, \ldots, N_{p}
\end{aligned}
$$

where $p_{i}^{\text {min }}$ and $p_{i}^{\max }$ are the lower and upper bounds of each parameter, respectively. As pointed out above, the problem (12) is set for a single train speed $v$, which simplifies the calculations but means that the optimal geometry obtained needs to be checked for other train speeds.

Note that in this work in order to explore new catenary configurations some combination of parameters $\mathbf{p}$ resulted in non-desirable catenaries from a practical point of view. Only the following restrictions were taken into account:

- Contact losses were not allowed, whereby the interaction force must be positive at any time $t$.

- All the droppers must be in tension in the static equilibrium configuration. If certain dropper $d$ is slackened in the static equilibrium position, this catenary is no longer valid.

Generally, two main groups of solvers are suitable for solving an optimisation problem: those based on the gradient and the so-called Genetic Algorithms (GA). The latter group of solvers was chosen in this work because a gradient-based method would require a sensitivity analysis of the objective function with respect to the parameters, which is not feasible for this problem. GAs try to reproduce the stochastic process of natural selection, obtaining the global optimum even for non-linear or discontinuous objective functions, which makes them a very attractive option.

The GA used in the present study is the one included in MATLAB ${ }^{\circledR}$ software. For a problem of $N_{p}$ optimisation variables (for example $N_{p}$ dropper lengths), the population size $n$ is chosen (i.e. $n$ different combinations of dropper lengths). The variables are taken as discrete in order to make a finite size space of variables (length increments of $1 \mathrm{~mm}$ are considered). Any individual which fulfils one of the two conditions listed above is 
excluded from the population.

An initial population evolves towards better solutions from generation to generation following the principles of natural selection, crossover and mutation (see Fig.4). A stochastic uniform process of selection was selected. The three best-scored parents were considered as elite and were moved directly to the next generation. A crossover fraction of 0.8 has been set, which means that $80 \%$ of the children came from a random combination of parent parameters. The rest of the children were randomly obtained by mutation of the parameters of a single parent.

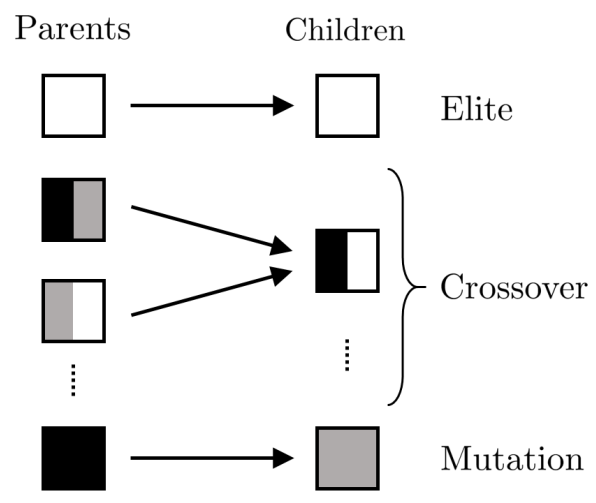

Figure 4: Scheme of the next generation creation process.

The algorithm runs until a certain stop criterion is accomplished. Specifically, the calculations stop when the average cumulative change in value of the objective function over a certain number of generations is less than a prescribed tolerance.

\section{Numerical results}

The numerical results presented in this work were obtained from two different catenary models, which are depicted in Fig. 5. The first catenary has a rather simple topology and was thoroughly described in the Benchmark [14] along with the pantograph model associated with it. Unlike this model, the second catenary has stitch wires (SW) at the support locations. Both catenary models are composed of 20 spans. Their contact wire are held horizontally, with no initial sag. These models are used as reference catenaries for comparison with the results obtained. 


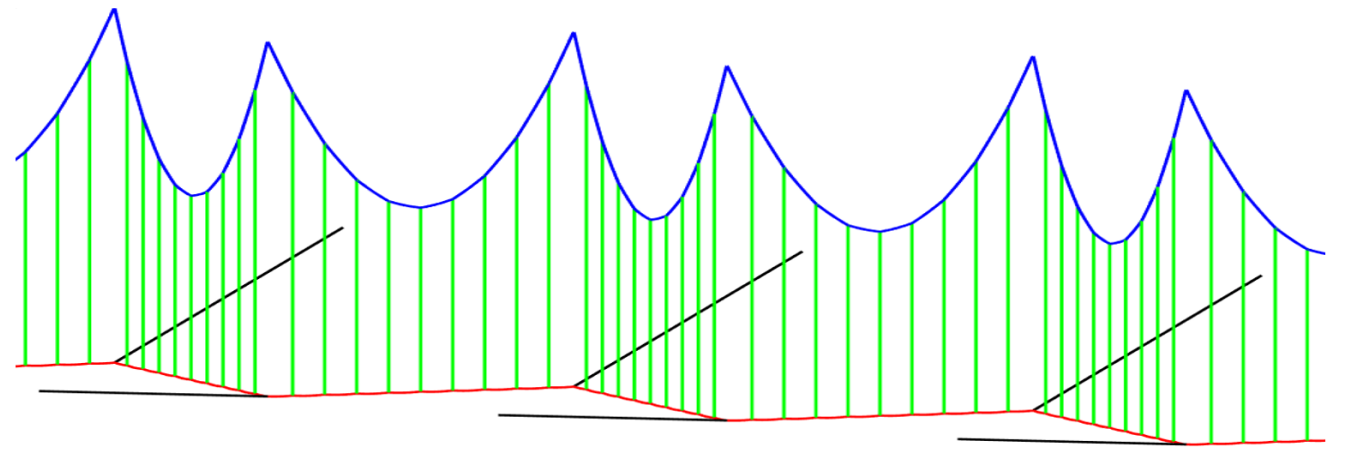

(a)

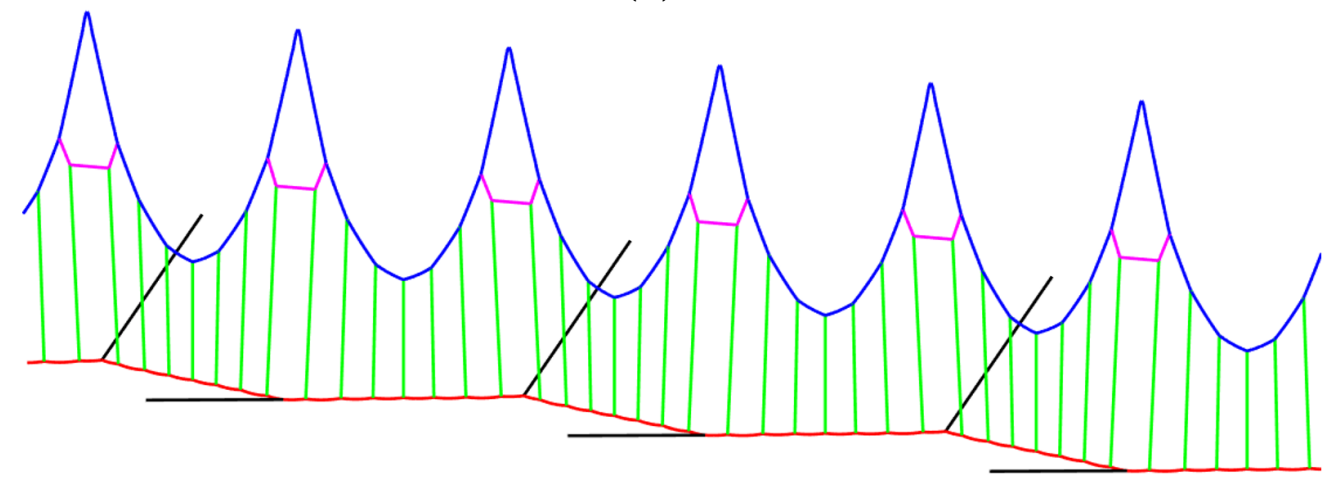

(b)

Figure 5: (a) Benchmark catenary and (b) SW catenary models.

All the dynamic simulations are carried out with a time step of $1 \mathrm{~ms}$ and the Newmark constants are set as $\gamma=0.5$ and $\beta=0.25$. The contact or interaction force is measured on the 10 central spans in order to avoid boundary effects. This force is also low-pass filtered to $20 \mathrm{~Hz}$. A velocity of $v=300 \mathrm{~km} / \mathrm{h}$ is considered to be the design train speed for both catenaries, which implies a mean contact interaction force $\bar{f}_{\text {inter }}=157.3 \mathrm{~N}$ in both cases. After performing a dynamic interaction simulation, the reference Benchmark catenary presents a $\sigma_{B}=32.99 \mathrm{~N}$, while the standard deviation of the interaction force for the SW catenary is $\sigma_{S W}=22.3 \mathrm{~N}$.

In what follows, Subsection 6.1 contains a test of the optimisation algorithm carried out by means of an optimisation of the pre-sag. Subsections 6.2 and 6.3 are devoted to find the best topologies of the Benchmark and the SW catenaries, respectively, i.e. the dropper lengths and the dropper spacing which provide the better dynamic behaviour for current collection quality purposes. Finally, in Subsection 6.4 the found optimal catenaries are analysed in terms of their static and dynamic behaviour. 


\subsection{Pre-sag optimisation}

The so-called pre-sag is the contact wire sag in the static equilibrium configuration. Presag is established in order to mitigate the difference in stiffness between the centre of the span and the supports. Several dynamic problems with different pre-sag were solved for both catenaries. As shown in Fig. 6, the amount of pre-sag strongly influences the standard deviation of the interaction force $\sigma$ in the 10 central spans. For a given train speed, large values of pre-sag and negative sags adversely affect the current collection quality for both the Benchmark (squares) and the SW (circles) catenaries.

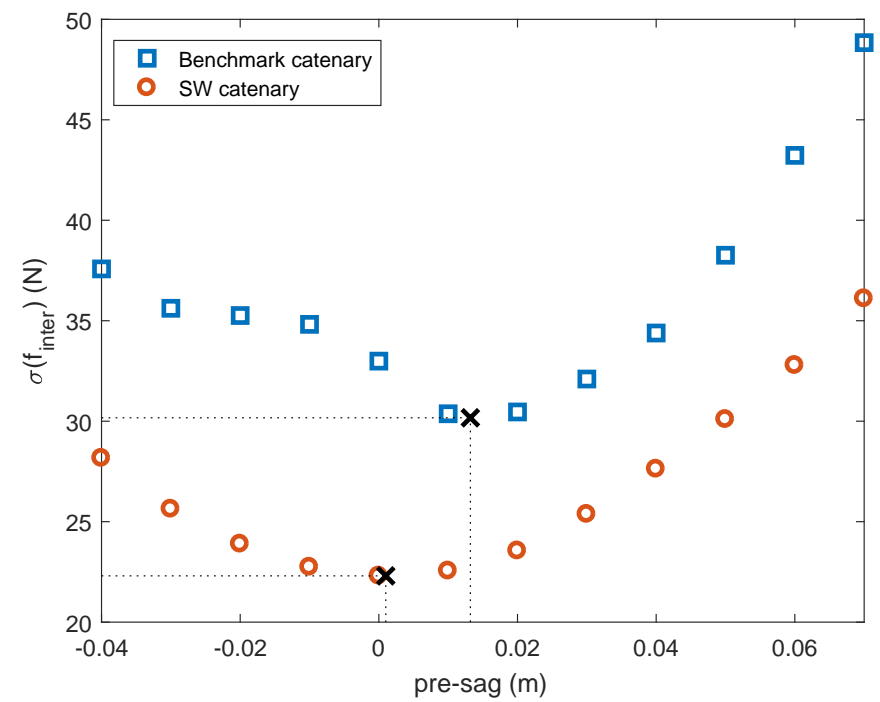

Figure 6: Influence of pre-sag for a train speed of $v=300 \mathrm{~km} / \mathrm{h}$.

In order to test the Genetic Algorithm (GA), an optimisation of the pre-sag at $v=300$ $\mathrm{km} / \mathrm{h}$ is carried out. In this academic example, there is only one optimisation variable, therefore only 8 generations with a population of 15 individuals are enough for the GA to find the minimum. Optimal pre-sag is highlighted for both catenaries in Fig. 6 by a cross, in which perfect agreement with the expected values is observed.

The optimal pre-sag is thought to be strongly affected by train speed. To investigate this issue, some optimisations are carried out at velocities ranging from 200 to $320 \mathrm{~km} / \mathrm{h}$. The optimal pre-sag (left) and the minimum obtained $\sigma$ (right) are shown in Fig. 7.

Regarding the smoother interaction force, it is clear that the SW catenary behaves better 


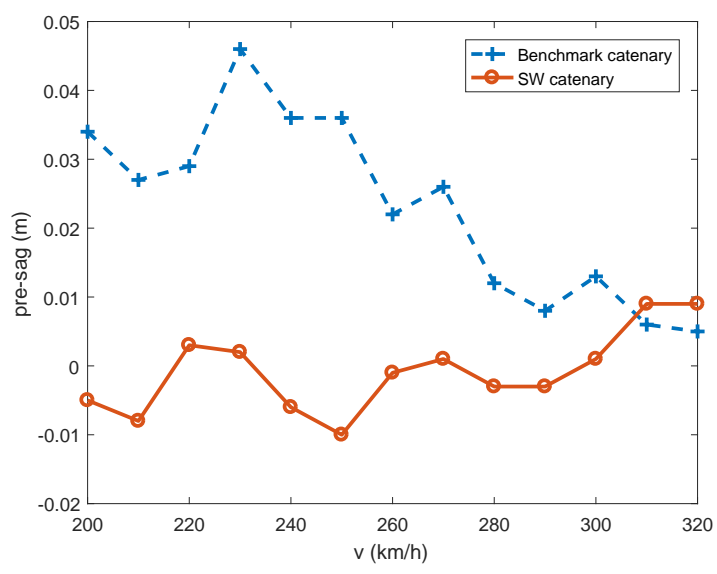

(a)

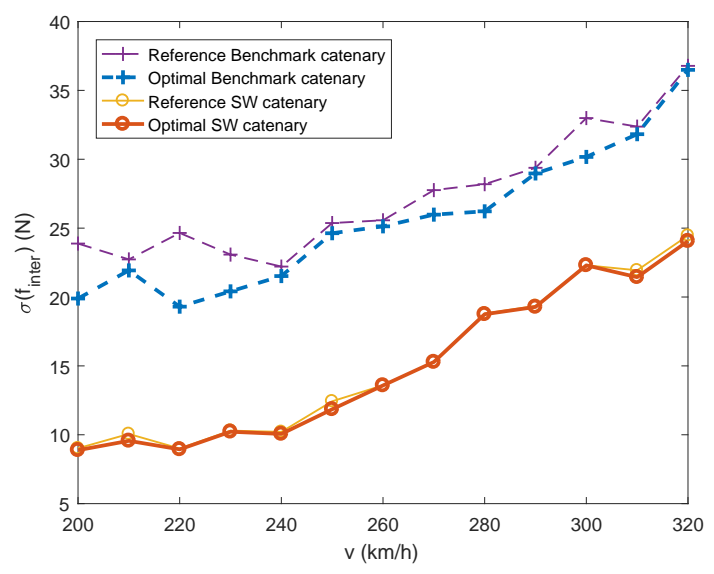

(b)

Figure 7: Evolution of the (a) optimal pre-sag and (b) minimum and reference contact force $\sigma$, with respect to the train speed.

than the Benchmark catenary for all the studied velocities, although both models cannot be compared since the Benchmark catenary is a model created for academic purposes only. However, for all the catenary models the interaction force shows higher variability, as evidenced by the increasing tendency of $\sigma$ as train speed increases. On the other hand, the optimal pre-sag shows different tendencies for each catenary type. While the optimal behaviour of the SW catenary is achieved with hardly any initial sag, for the Benchmark catenary, the lower the velocity the more beneficial pre-sag seems to be. According to the results, for this type of catenary a pre-sag between $1 / 1000$ and 1/2000 of span length is optimal at velocities below $270 \mathrm{~km} / \mathrm{h}$.

The optimal $\sigma$ can be also compared with respect to the ones obtained at the same train speeds for both reference catenaries, i.e. catenaries with no contact wire sag (see the thin curves in Fig. 7b). This comparison reveals that for the SW catenary the greatest reduction in $\sigma$ is only $5 \%$ at $v=210 \mathrm{~km} / \mathrm{h}$. This result confirms that with the presence of pre-sag there is no observable improvement in current collection quality for this catenary with stitch wires. Conversely, the Benchmark catenary shows a better improvement in the presence of pre-sag, especially for low train speeds. For example, at $v=220 \mathrm{~km} / \mathrm{h}$, $\sigma$ is reduced by $21.7 \%$. Despite this result, it seems to be reasonable to seek some more appealing variables for which the catenary system dynamic behaviour could be optimised. 


\subsection{Benchmark catenary optimisation}

The first optimisation carried out for the Benchmark catenary considers $\Delta h_{c_{i}}$ for $i=$ $1, \ldots, N_{p}$ as optimisation parameters. $\Delta h_{c_{i}}$ denotes the difference in height at dropper point $i$ from the reference catenary configuration, as seen in Fig. 8. These variables are intimately related with the length of the dropper connected at this point.

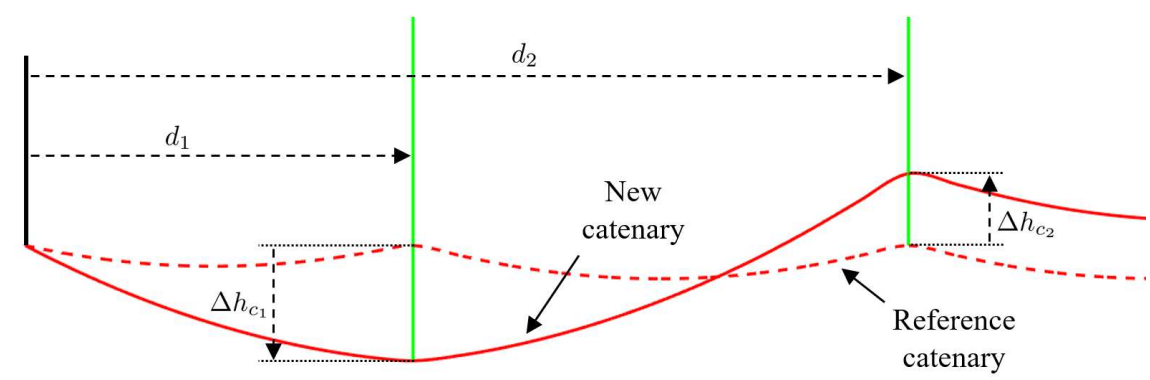

Figure 8: Graphical description of variables $\Delta h_{c_{i}}$.

Since every span must be equal and their symmetry has to be preserved, the number of variables of the problem amounts to $N_{p}=5$. The 5 variables ranges from $\Delta h_{c_{i}}^{\min }=-0.02 \mathrm{~m}$ to $\Delta h_{c_{i}}^{\max }=0.02 \mathrm{~m}$ at intervals of $1 \mathrm{~mm}$. This problem is labelled as B1, and the result shown in the first row of Table 1 is obtained after 120 generations with a population of 100 individuals.

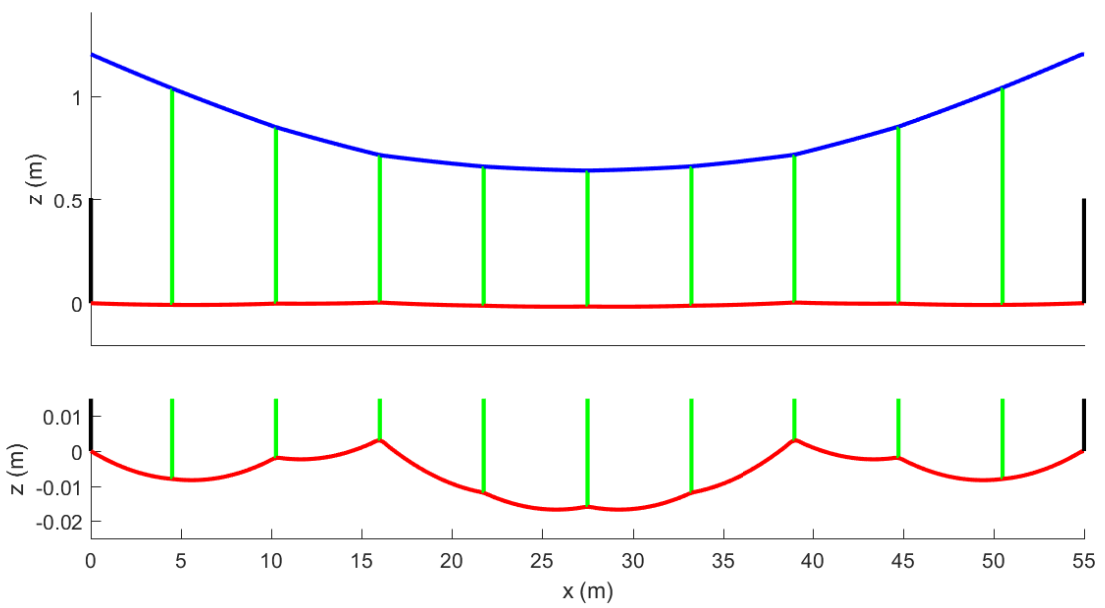

Figure 9: Optimal geometry obtained in problem B1.

A span of the B1 optimised catenary is depicted in Fig. 9, in which a non-uniform contact 
wire height is seen. It is important to highlight that with only small changes in dropper lengths, the optimal configuration obtained produces a $\sigma_{B 1}=17.71 \mathrm{~N}$, i.e. more than $46 \%$ less than that of the reference catenary with a horizontal contact wire.

\begin{tabular}{ccccc}
\hline $\begin{array}{c}\text { Problem } \\
\text { identifier }\end{array}$ & $\begin{array}{c}\text { Variable } \\
\text { type }\end{array}$ & Optimal values $(\mathrm{m})$ & $\begin{array}{c}\sigma\left(f_{\text {inter }}\right) \\
(\mathrm{N})\end{array}$ & $\begin{array}{c}\sigma \text { reduction } \\
(\%)\end{array}$ \\
\hline B1 & $\Delta h_{c}$ & $-0.008-0.0020 .003-0.012-0.016$ & 17.71 & 46.32 \\
B2 & $d$ & 7.39 .415 .826 .4 & 22.8 & 30.89 \\
B3 & $d, \Delta h_{c}$ & $9.815 .8,-0.0020 .001-0.018$ & 18.44 & 44.10 \\
\hline
\end{tabular}

Table 1: Optimisation results of the Benchmark catenary.

As mentioned above, the Benchmark catenary has droppers equally spaced within a span. The second optimisation problem, labelled B2, is intended to find the optimal dropper spacing for the design speed, while no sag is applied to the contact wire. In this case, the $x$ absolute position $d_{i}$ for $i=1, \ldots, N_{p}$, are the optimisation variables. To preserve the symmetry of the span, the central dropper cannot be moved, therefore $N_{p}=4$. In order to avoid overlapping and trying to find a dropper distribution as uniform as possible, the proposed range for each variable is shown in Table 2. These domains, which notably reduce the optimisation variables' space, are discretised into increments of $1 \mathrm{~cm}$ in length.

\begin{tabular}{ccccc}
\hline$i$ & 1 & 2 & 3 & 4 \\
\hline$d_{i}^{\text {min }}(\mathrm{m})$ & 0.1 & 7.5 & 13.2 & 18.9 \\
$d_{i}^{\text {max }}(\mathrm{m})$ & 7.4 & 13.1 & 18.8 & 27.4 \\
\hline
\end{tabular}

Table 2: Variable limits used in the B2 optimisation problem.

The GA stopped after 85 generations, composed of 80 individuals each, giving the results shown in Table 1. A graphical representation of the optimal B2 catenary geometry is given in Fig. 10. In this case $\sigma_{B 2}=22.8 \mathrm{~N}$, which represents almost a $31 \%$ reduction when compared to the reference Benchmark catenary. Although this reduction is not as large as the one obtained in the B1 optimisation, dropper spacing undoubtedly arises as a key factor in improving current collection quality.

A deeper study of the B2 catenary configuration reveals that the first and second droppers are placed very close to each other and that there is a cluster of three droppers in the middle of the span. This suggests that there could be redundant droppers within the span and some could be eliminated without major effects on the dynamic behaviour. In this case, a span composed of 5 droppers seems to be a reasonable option. 


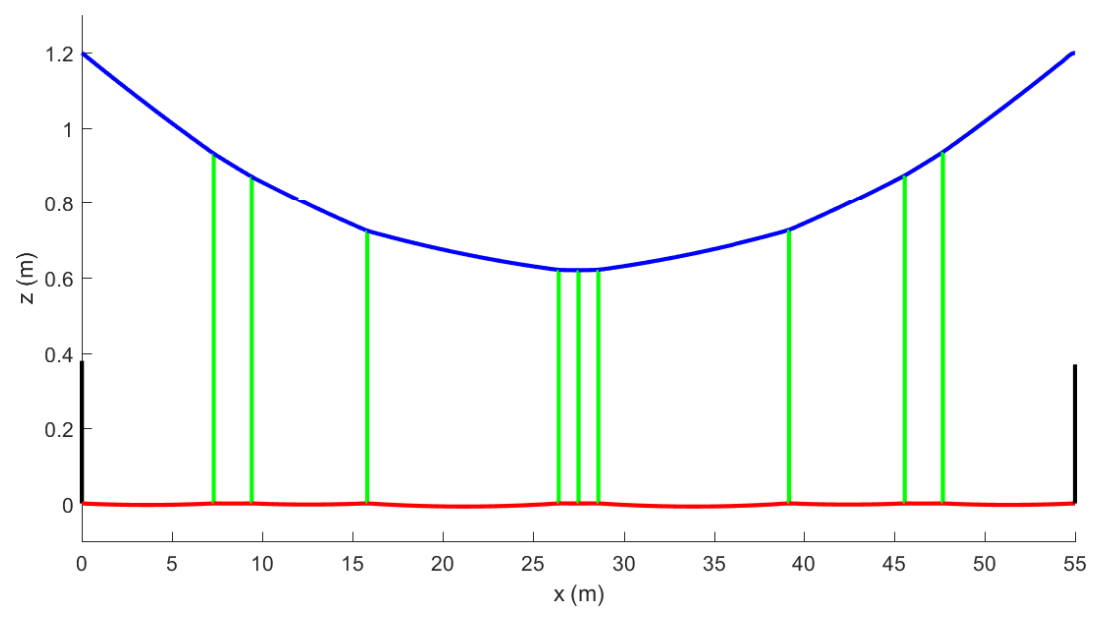

Figure 10: Optimal geometry obtained in problem B2.

For this new catenary with fewer droppers the B3 optimisation is carried out, consisting of optimising both dropper lengths, by means of the height of the dropper connection point to the contact wire, and dropper spacing. There are 5 optimisation variables altogether, 3 heights and 2 horizontal dropper locations. The size of the population is set to 100 individuals and the limits for each variable are listed in Table 3.

\begin{tabular}{cccccc}
\hline & \multicolumn{2}{c}{$d_{i}(\mathrm{~m})$} & \multicolumn{3}{c}{$\Delta h_{c_{i}}(\mathrm{~m})$} \\
\hline$i$ & 1 & 2 & 1 & 2 & 3 \\
\hline Min. & 0.1 & 13.1 & -0.02 & -0.02 & -0.02 \\
Max. & 13 & 27.4 & 0.02 & 0.02 & 0.02 \\
\hline
\end{tabular}

Table 3: Variable limits used in the B3 optimisation problem.

After 70 generations the optimisation problem is solved, giving the optimal variables that appear in Table 1. With 4 fewer droppers per span, the optimal B3 catenary, depicted in Fig. 11, offers almost the same good behaviour as the B1 topology in terms of $\sigma$. For this problem, the optimal solution presents a $\sigma_{B 3}=18.44 \mathrm{~N}$, which is $44 \%$ lower than that obtained with the reference catenary.

\subsection{Stitch wired catenary optimisation}

In this section, the topology of the catenary with stitch wires is optimised. In the first place, dropper length optimisation is carried out, in which $\Delta h_{c_{i}}$, for $i=1, \ldots, N_{p}$, are 


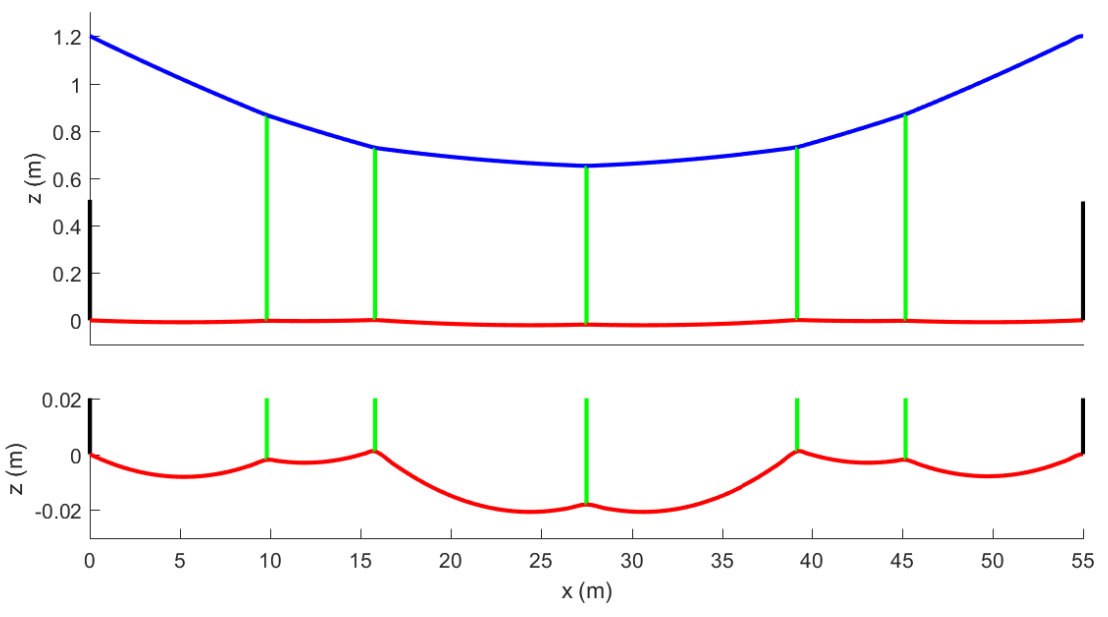

Figure 11: Optimal geometry obtained in problem B3.

considered as optimisation variables. Again, as every span must be equal and symmetric, the number of variables in this problem is $N_{p}=4$. The range of variation allowed for the 4 variables is $\Delta h_{c_{i}}^{\text {min }}=-0.02 \mathrm{~m}$ to $\Delta h_{c_{i}}^{\max }=0.02 \mathrm{~m}$, as in the B1 optimisation. This problem is labelled SW1, and the optimal solution is obtained after 90 generations with a population size of 80 .

The result of the SW1 problem is given in Table 5 and Fig. 12. The SW1 catenary undergoes a smoother interaction force, as is clear from $\sigma_{S W 1}=14.14 \mathrm{~N}$, than the reference stitch wire catenary, with which a $36.6 \%$ greater $\sigma$ is obtained. Comparing the SW1 configuration with the B1 catenary (see Fig. 9), the main difference is in the height of the central point, which is much lower for the Benchmark catenary type.

The second optimisation concerns the search for the optimal dropper spacing in terms of current collection quality. For this case there are only 3 optimisation variables $d_{i}$, since the dropper located at midspan cannot be moved to preserve the symmetry. This problem is labelled SW2 and the allowed ranges for each variable are shown in Table 4.

\begin{tabular}{cccc}
\hline$i$ & 1 & 2 & 3 \\
\hline$d_{i}^{\min }(\mathrm{m})$ & 0.1 & 9.1 & 20.5 \\
$d_{i}^{\max }(\mathrm{m})$ & 8.9 & 20.4 & 32.4 \\
\hline
\end{tabular}

Table 4: Variable limits used in the SW2 optimisation problem.

With a population of 60 individuals, after 54 generations the GA found the optimal 

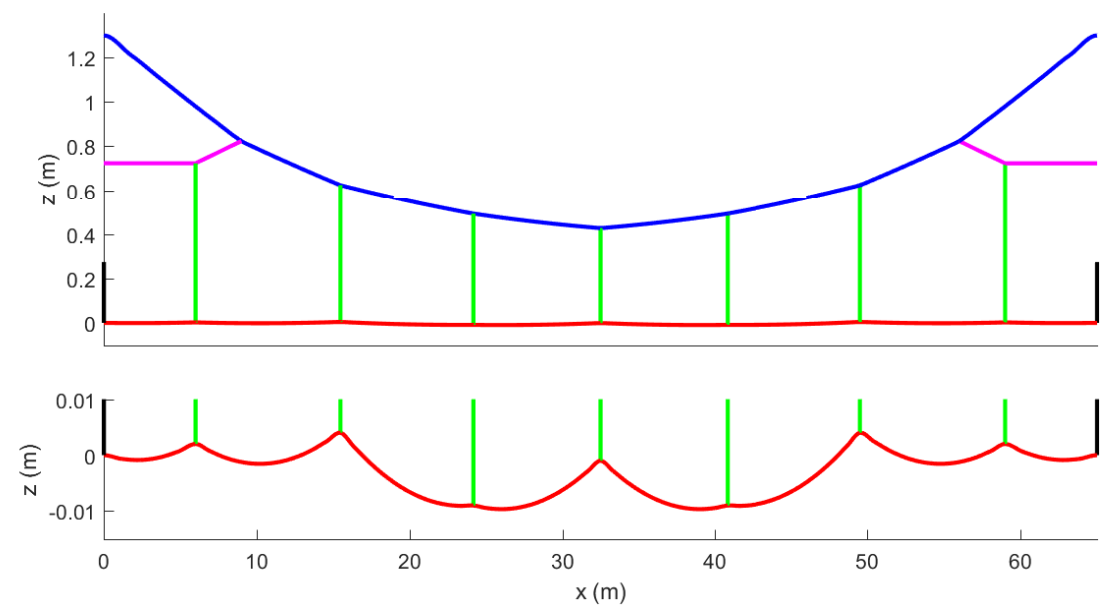

Figure 12: Optimal geometry obtained in SW1 problem.

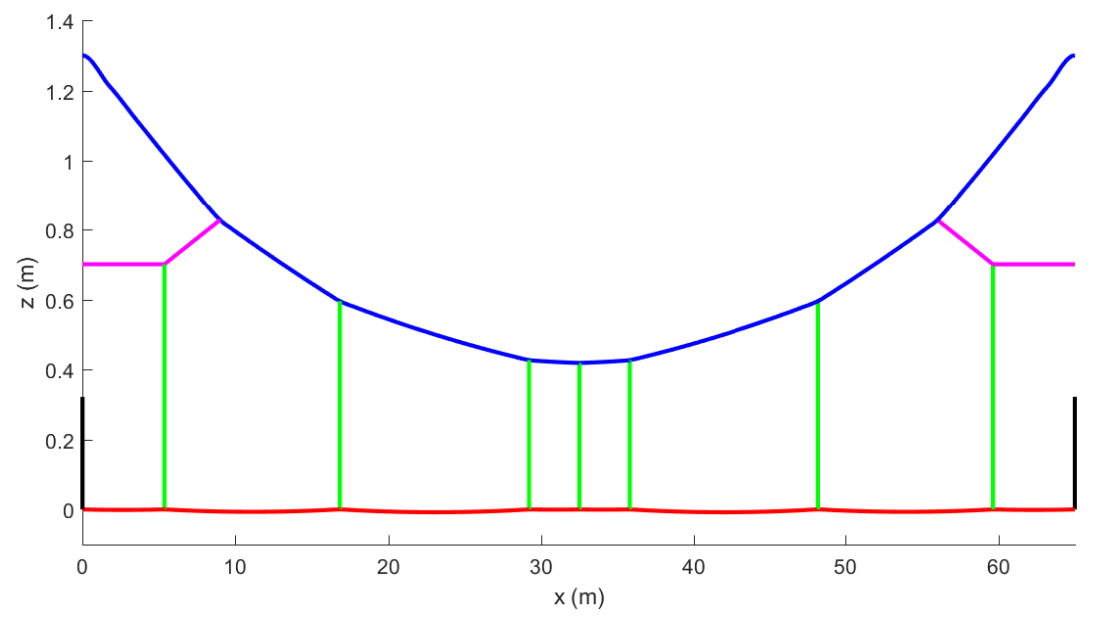

Figure 13: Optimal geometry obtained in SW2 problem.

solution, which can be seen in Table 5. In Fig. 13 a span of the optimal SW2 catenary is plotted. The standard deviation of the interaction force is very similar to that achieved by the SW1 configuration. Specifically, $\sigma\left(f_{\text {inter }}\right)=14.05 \mathrm{~N}$.

\begin{tabular}{ccccc}
\hline $\begin{array}{c}\text { Problem } \\
\text { identifier }\end{array}$ & $\begin{array}{c}\text { Variable } \\
\text { type }\end{array}$ & Optimal values $(\mathrm{m})$ & $\begin{array}{c}\sigma\left(f_{\text {inter }}\right) \\
(\mathrm{N})\end{array}$ & $\begin{array}{c}\sigma \text { reduction } \\
(\%)\end{array}$ \\
\hline SW1 & $\Delta h_{c}$ & $0.0020 .004-0.009-0.001$ & 14.14 & 36.60 \\
SW2 & $d$ & 5.3616 .8329 .21 & 14.05 & 44.22 \\
SW3 & $d, \Delta h_{c}$ & $6.0418 .27,0.0020 .0040$ & 12.42 & 44.31 \\
\hline
\end{tabular}

Table 5: Optimisation results of the $S W$ catenary. 
The location of the three droppers near the midspan indicates the possibility of removing two of them, so that the SW3 optimisation problem is carried out on a SW catenary with only 5 droppers per span. Like the B3 problem, in this case there are 5 optimisation variables composed of 2 dropper horizontal positions and 3 heights of the contact wire points. Their ranges are listed in Table 6 . Since there are 5 variables to optimise, a population of 100 individuals is set.

\begin{tabular}{cccccc}
\hline & $d_{i}(\mathrm{~m})$ & \multicolumn{3}{c}{$\Delta h_{c_{i}}(\mathrm{~m})$} \\
\hline$i$ & 1 & 2 & 1 & 2 & 3 \\
\hline Min. & 2 & 10 & -0.02 & -0.02 & -0.02 \\
Max. & 9 & 30 & 0.02 & 0.02 & 0.02 \\
\hline
\end{tabular}

Table 6: Variable limits used in the $S W 3$ optimisation problem.

The GA only took 85 generations to find the optimal solution shown in Table 5. In this catenary topology the three central droppers are almost equally spaced, as can be seen in Fig. 14. The dispersion of the interaction force is the lowest of the three optimised SW catenaries, with $\sigma_{S W 3}=12.42 \mathrm{~N}$. This represents a reduction of $44.31 \%$ with respect to the value obtained for the reference SW catenary.

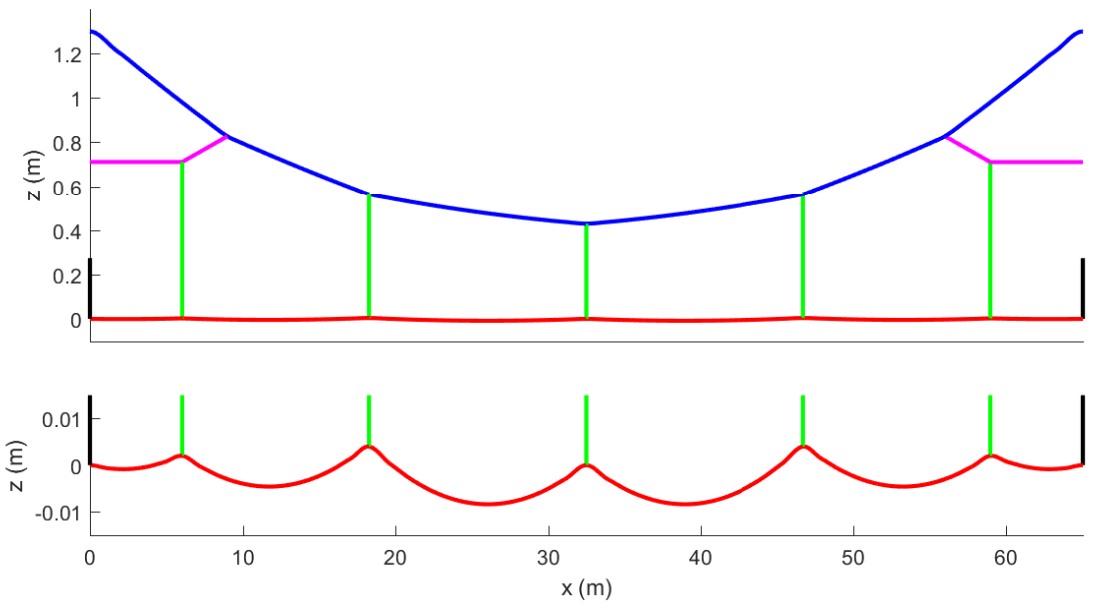

Figure 14: Optimal geometry obtained in $S W 3$ problem. 


\subsection{Analysis of the optimal catenaries}

It is interesting to analyse some static and dynamic characteristics of the optimal catenary configurations obtained in the previous sections. Specifically, the stiffness of the catenary along with the internal force of the droppers in the static equilibrium position are explored and the behaviour of the optimal catenaries at train speeds different to the design speed is also investigated.

\subsubsection{Static characteristics of the optimal catenaries}

The stiffness $k_{s t}$ at a certain point in the catenary is defined as the relationship between the vertical uplift and the upward force applied thereon. Fig. 15 and Fig. 16 show this magnitude calculated in a central span subjected to a load $F=100 \mathrm{~N}$ (left) or $F=200 \mathrm{~N}$ (right) for the Benchmark and the Stitch Wired catenaries respectively. Particularly for the former, a noticeable difference in stiffness is observed when the value of the applied force changes. This is due to the unilateral non-linearity exhibited by the droppers, which tend to slacken and make the catenary more flexible.
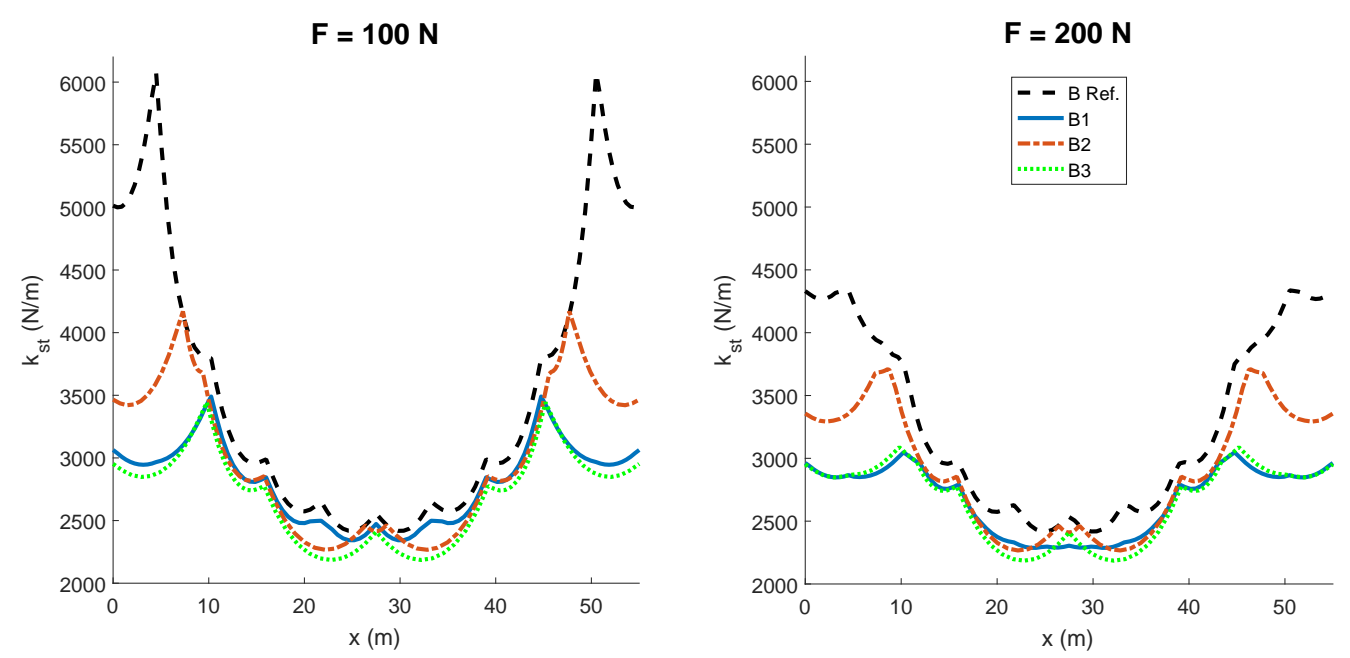

Figure 15: Vertical stiffness of the Benchmark catenaries.

Comparing the optimal catenary configurations with respect to their reference configura- 

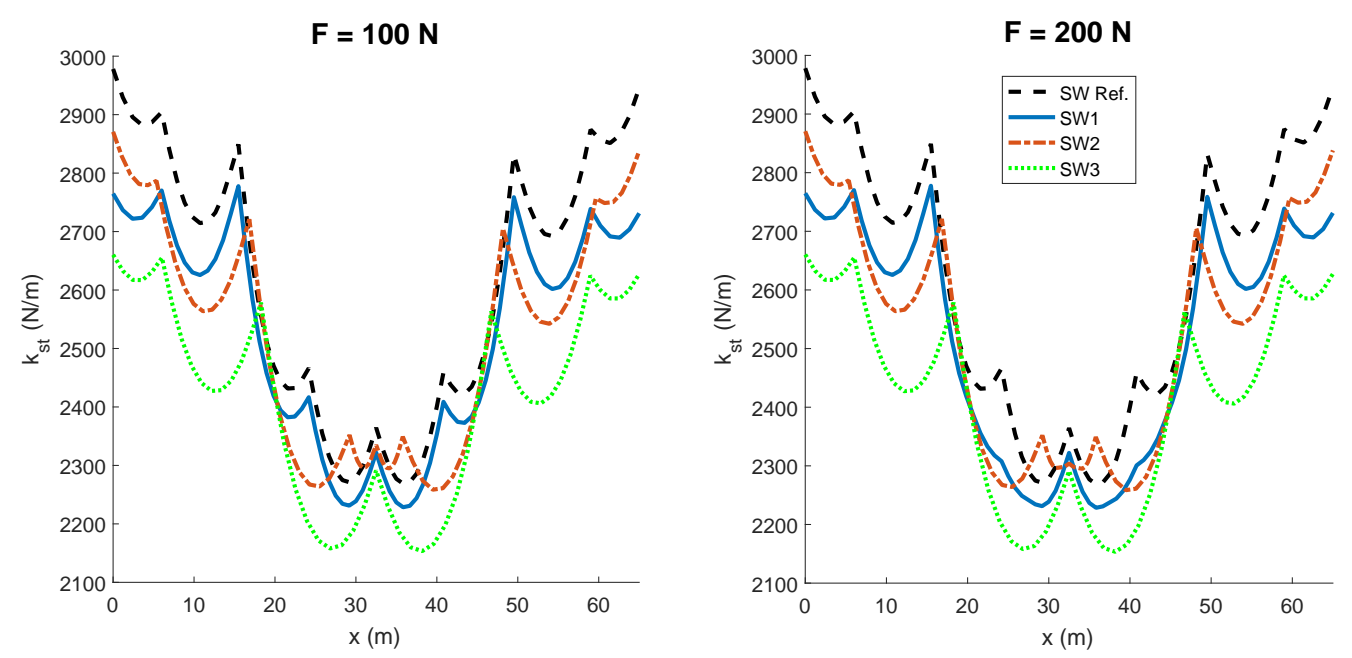

Figure 16: Vertical stiffness of the $S W$ catenaries.

tions, in both Benchmark and SW catenaries there is a reduction in the variability of the stiffness along the span. This means a smaller difference between the maximum $k_{s t}^{\max }$ and the minimum $k_{s t}^{\max }$ stiffness values. In order to quantify this effect, the stiffness variability coefficient is defined as:

$$
\alpha=\frac{k_{s t}^{\max }-k_{s t}^{\min }}{k_{s t}^{\max }+k_{s t}^{\min }}
$$

This coefficient is calculated for all the eight catenaries, as shown in Table 7. A large decrease of the stiffness variability is noticed in the optimal Benchmark catenaries if compared with the reference configuration. For catenary B1, this reduction exceeds $50 \%$. In contrast, the smoothing of the stiffness is limited to $23 \%$ greater than the reference case in SW catenaries. What emerges from these results is the benefit for current collection quality of having a more uniform catenary stiffness distribution at the train speed studied.

\begin{tabular}{ccccc}
\hline Cat. Label & $F=100 \mathrm{~N}$ & Reduction $(\%)$ & $F=200 \mathrm{~N}$ & Reduction (\%) \\
\hline B Ref. & 0.430 & - & 0.284 & - \\
B1 & 0.197 & 54.19 & 0.143 & 49.65 \\
B2 & 0.296 & 31.16 & 0.241 & 15.14 \\
B3 & 0.223 & 48.14 & 0.171 & 39.79 \\
\hline SW Ref. & 0.135 & - & 0.135 & - \\
SW1 & 0.109 & 19.26 & 0.109 & 19.26 \\
SW2 & 0.118 & 12.59 & 0.118 & 12.59 \\
SW3 & 0.104 & 22.96 & 0.104 & 22.96 \\
\hline
\end{tabular}

Table 7: Stiffness variation coefficient $\alpha$ of the optimal catenaries. 
Another aspect that requires to be analysed is the internal force of droppers $f_{d}$ in the static equilibrium configuration. In Fig. 17 these forces are represented for the Benchmark reference and optimal catenaries. For the reference configuration, with no sag, all the droppers are uniformly tensioned, whilst large differences in dropper tensions are present for optimised Benchmark catenaries. It is observed that certain droppers, such as the $1^{\text {st }}$ and $9^{\text {th }}$ droppers in B1 or the central dropper in B2, are barely loaded. A very similar scenario is found for SW catenaries, as shown in Fig. 18.
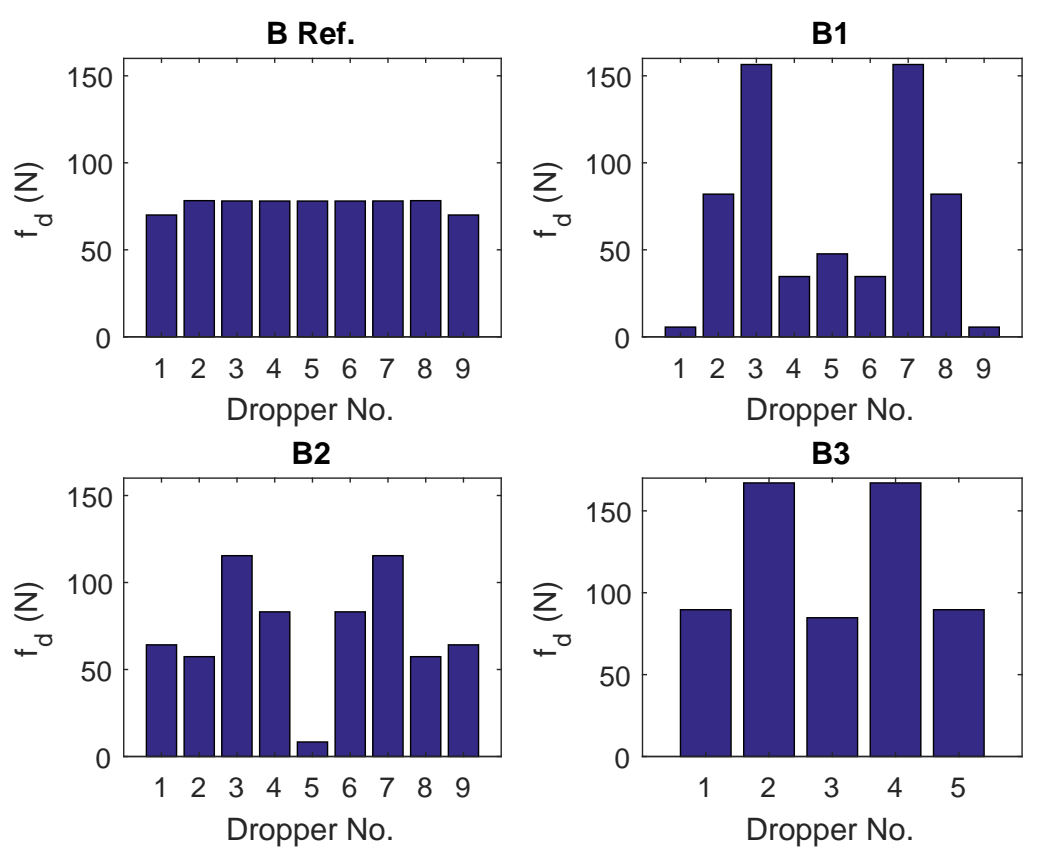

Figure 17: Dropper forces in static equilibrium configuration for Benchmark catenaries.

\subsubsection{Dynamic behaviour of the optimal catenaries}

As pointed out in Section 5, catenary optimisations are carried out only for a single train speed, which means the optimal catenaries must be tested at other train velocities. Fig. 19 shows the standard deviation $\sigma$ of the interaction force for B1, B2 and B3 optimal configurations at different speeds. These results are also compared to those of the reference catenary for the same range of velocities.

Fig. 19 reveals that catenaries B1 and B3 behave similarly when train speed changes. Their interaction force when the train travels at velocities near the design speed is much 

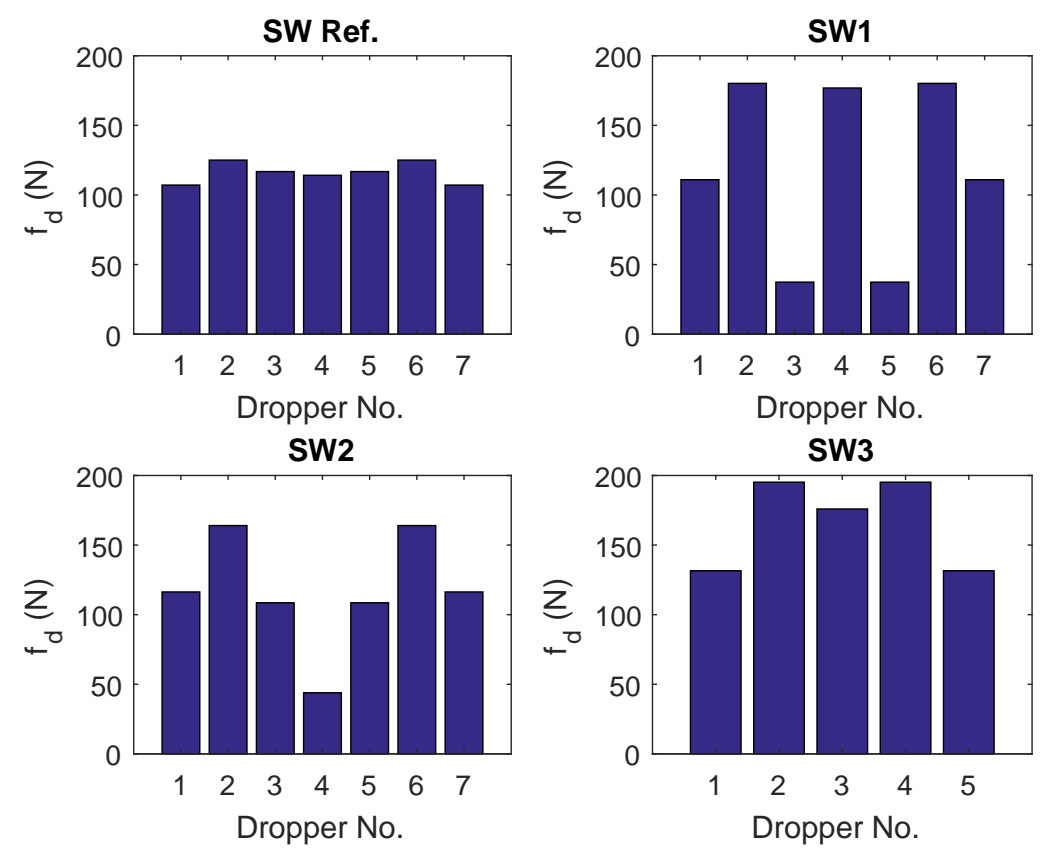

Figure 18: Dropper forces in static equilibrium configuration for $S W$ catenaries.

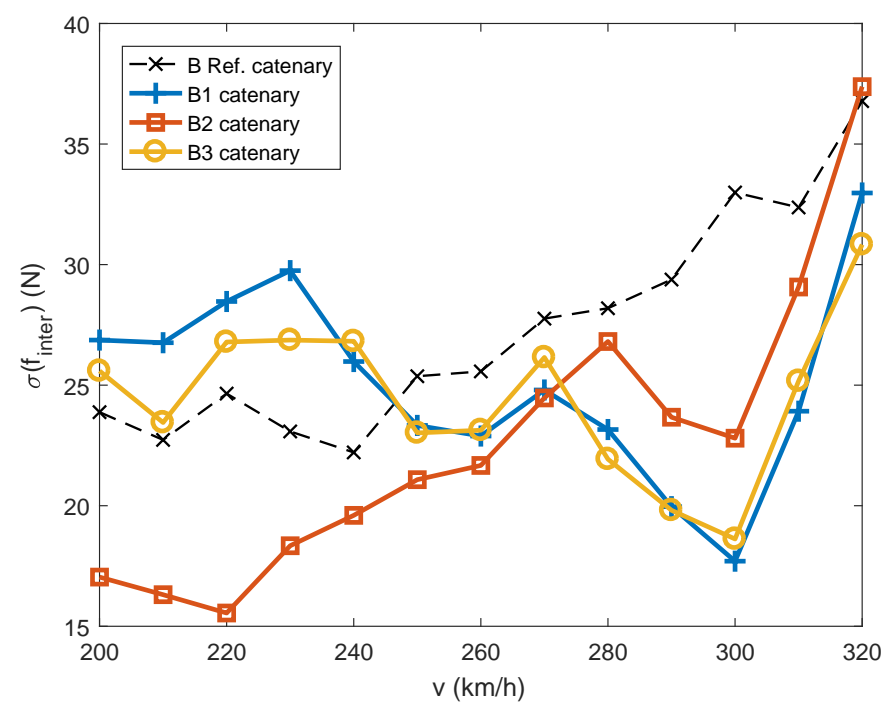

Figure 19: Optimised Benchmark catenaries behaviour at different train speeds.

less oscillatory than that of the reference catenary. However, at velocities below $250 \mathrm{~km} / \mathrm{h}$, configurations B1 and B3 show large oscillations of the interaction force, which is not a desirable effect. On the other hand, catenary B2 has very good performance for almost the entire speed range analysed. This means that, although this topology is not as good 
as the B1 and the B3 at the design speed, it has the best behaviour when the overall speed range is taken into account.

Moving to the optimal SW catenary configurations, Fig. 20 shows their dynamic behaviour, for a wide range of train speeds, in terms of $\sigma$. In this case, the catenary with optimal dropper lengths (SW1) has a highly oscillatory interaction force for velocities below the design speed. This large $\sigma$ is also highly dependent on train speed. However, catenaries SW2 and SW3 show good behaviour at low train speeds, although not as good as the reference SW catenary. Unlike catenary SW1, these two options seem to be more stable versus velocity changes, with SW3 the most suitable solution according to this criterion. In addition, the SW3 catenary presents the lowest interaction force $\sigma$ at the design train speed.

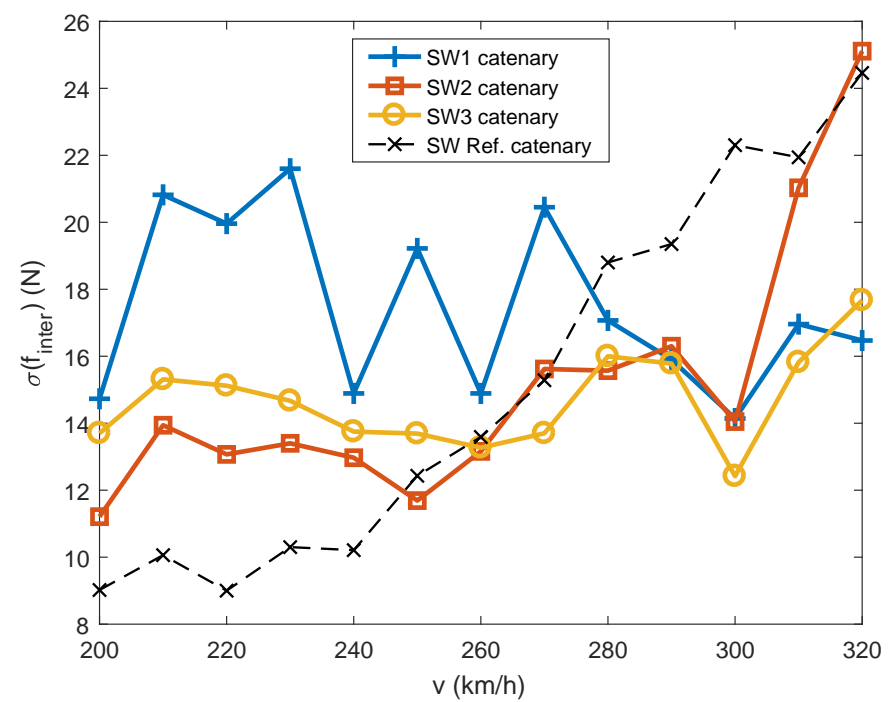

Figure 20: Optimised $S W$ catenaries' behaviour at different train speeds.

\section{Conclusions}

This paper describes an attempt to find the optimal catenary configuration in terms of current collection quality. This optimisation consists of finding the catenary geometry which leads to the minimal standard deviation of the pantograph-catenary interaction force. The optimisations were carried out by means of a Genetic Algorithm in which 
dropper lengths, dropper spacing, or both, were set as optimisation variables.

Several optimisations were carried out for two different catenary types: with and without stitch wires. The results of the optimisations show that current collection quality can be hugely improved with only slight variations in dropper lengths, that great benefits can be obtained by varying dropper spacing and that even the removal of certain droppers can be considered.

In the static configurations the optimal catenaries show more uniform stiffness and conversely lower uniformity in dropper forces than their respective reference catenary geometries. As regards the dynamic behaviour at different train speeds, the catenaries with optimal dropper spacing seem to be the most consistent options. Although they are not the best ones at the design speed, their behaviour at other velocities does not get worse or may be even better than the reference catenaries.

In conclusion, the results show that not only pre-sag can be a beneficial factor in current collection, but that catenary designers should also consider other geometric parameters such as contact wire height distribution or dropper spacing within the span, because they can lead to greater reductions in interaction force fluctuations. Besides, the GA tool can be used to good effect in designing better catenaries.

\section{Acknowledgements}

The authors would like to acknowledge the financial support received from the FPU program offered by the Ministry of Education, Culture and Sports of Spain (MECD), under grant number FPU13/04191, and also the funding provided by the Regional Government of Valencia (PROMETEO/2016/007).

\section{References}

[1] P. Nåvik, A. Rønnquist, and S. Stichel, "The use of dynamic response to evaluate and improve the optimization of existing soft railway catenary systems for higher 
speeds," Proceedings of the Institution of Mechanical Engineers, Part F: Journal of Rail and Rapid Transit, vol. 230, no. 4, pp. 1388-1396, 2016.

[2] P. Harell, L. Drugge, and M. Reijm, "Study of critical sections in catenary systems during multiple pantograph operation," Proceedings of the Institution of Mechanical Engineers, Part F: Journal of Rail and Rapid Transit, vol. 219, no. 4, pp. 203-211, 2005.

[3] Y. H. Cho, "SPOPS statement of methods," Vehicle System Dynamics, vol. 53, no. 3, pp. 329-340, 2015.

[4] C. Sánchez-Rebollo, A. Carnicero, and J. R. Jiménez-Octavio, "CANDY statement of methods," Vehicle System Dynamics, vol. 53, no. 3, pp. 392-401, 2015.

[5] M. Ikeda, “Gasen-do FE' statement of methods," Vehicle System Dynamics, vol. 53, no. 3, pp. 357-369, 2015.

[6] J.-P. Massat, E. Balmes, J.-P. Bianchi, and G. Van Kalsbeek, "OSCAR statement of methods," Vehicle System Dynamics, vol. 53, no. 3, pp. 370-379, 2015.

[7] A. Collina, S. Bruni, A. Facchinetti, and A. Zuin, "PCaDA statement of methods," Vehicle System Dynamics, vol. 53, no. 3, pp. 347-356, 2015.

[8] P.-A. Jönsson, S. Stichel, and C. Nilsson, "CaPaSIM statement of methods," Vehicle System Dynamics, vol. 53, no. 3, pp. 341-346, 2015.

[9] M. Tur, L. Baeza, F. Fuenmayor, and E. García, "PACDIN statement of methods," Vehicle System Dynamics, vol. 53, no. 3, pp. 402-411, 2015.

[10] J. Ambrósio, J. Pombo, P. Antunes, and M. Pereira, "PantoCat statement of methods," Vehicle System Dynamics, vol. 53, no. 3, pp. 314-328, 2015.

[11] A. Facchinetti and S. Bruni, "Special issue on the pantograph-catenary interaction benchmark," Vehicle System Dynamics, vol. 53, no. 3, pp. 303-304, 2015.

[12] L. Finner, G. Poetsch, B. Sarnes, and M. Kolbe, "Program for catenary-pantograph analysis, PrOSA statement of methods and validation according en 50318," Vehicle System Dynamics, vol. 53, no. 3, pp. 305-313, 2015. 
[13] N. Zhou, Q. Lv, Y. Yang, and W. Zhang, "TPL-PCRUN statement of methods," Vehicle System Dynamics, vol. 53, no. 3, pp. 380-391, 2015.

[14] S. Bruni, J. Ambrosio, A. Carnicero, Y. H. Cho, L. Finner, M. Ikeda, S. Y. Kwon, J.P. Massat, S. Stichel, and M. Tur, "The results of the pantograph-catenary interaction benchmark," Vehicle System Dynamics, vol. 53, no. 3, pp. 412-435, 2015.

[15] N. Zhou and W. Zhang, "Investigation on dynamic performance and parameter optimization design of pantograph and catenary system," Finite Elements in Analysis and Design, vol. 47, no. 3, pp. 288-295, 2011.

[16] J.-W. Kim and S.-N. Yu, "Design variable optimization for pantograph system of high-speed train using robust design technique," International Journal of Precision Engineering and Manufacturing, vol. 14, no. 2, pp. 267-273, 2013.

[17] J. Ambrósio, J. Pombo, and M. Pereira, "Optimization of high-speed railway pantographs for improving pantograph-catenary contact," Theoretical and Applied Mechanics Letters, vol. 3, no. 1, p. 013006, 2013.

[18] A. Bobillot, J. Massat, and J. Mentel, "Design of pantograph-catenary systems by simulation," in 9th World Congress on Railway Research (WCRR), 2011.

[19] J.-P. Massat, C. Laurent, J.-P. Bianchi, and E. Balmès, "Pantograph catenary dynamic optimisation based on advanced multibody and finite element co-simulation tools," Vehicle System Dynamics, vol. 52, no. sup1, pp. 338-354, 2014.

[20] J.-W. Kim, H.-C. Chae, B.-S. Park, S.-Y. Lee, C.-S. Han, and J.-H. Jang, "State sensitivity analysis of the pantograph system for a high-speed rail vehicle considering span length and static uplift force," Journal of sound and vibration, vol. 303, no. 3, pp. 405-427, 2007.

[21] Y. H. Cho, K. Lee, Y. Park, B. Kang, and K.-n. Kim, "Influence of contact wire pre-sag on the dynamics of pantograph-railway catenary," International journal of mechanical sciences, vol. 52, no. 11, pp. 1471-1490, 2010.

[22] W. Zhang, G. Mei, and J. Zeng, "A study of pantograph/catenary system dynamics with influence of presag and irregularity of contact wire," Vehicle System Dynamics, vol. 37, no. sup1, pp. 593-604, 2002. 
[23] C. Nordstrøm Jensen and H. True, "Dynamics of an electrical overhead line system and moving pantographs," Vehicle System Dynamics, vol. 29, no. S1, pp. 104-113, 1998.

[24] M. Tur, E. García, L. Baeza, and F. Fuenmayor, "A 3D absolute nodal coordinate finite element model to compute the initial configuration of a railway catenary," Engineering Structures, vol. 71, pp. 234-243, 2014.

[25] S. Gregori, M. Tur, E. Nadal, J. Aguado, F. Fuenmayor, and F. Chinesta, "Fast simulation of the pantograph-catenary dynamic interaction," Finite Elements in Analysis and Design, vol. 129, pp. 1-13, 2017.

[26] S. H. Kia, F. Bartolini, A. Mpanda-Mabwe, and R. Ceschi, "Pantograph-catenary interaction model comparison," in IECON 2010-36th Annual Conference on IEEE Industrial Electronics Society, pp. 1584-1589, IEEE, 2010.

[27] A. Shabana, "Computer implementation of the absolute nodal coordinate formulation for flexible multibody dynamics," Nonlinear Dynamics, vol. 16, pp. 293-306, 1998.

[28] J. Gerstmayr and A. A. Shabana, "Analysis of thin beams and cables using the absolute nodal co-ordinate formulation," Nonlinear Dynamics, vol. 45, no. 1-2, pp. 109$130,2006$.

[29] A. Collina and S. Bruni, "Numerical simulation of pantograph-overhead equipment interaction," Vehicle System Dynamics, vol. 38, no. 4, pp. 261-291, 2002.

[30] EN 50367, "Railway applications. Current collection systems. Technical criteria for the interaction between pantograph and overhead line," European Committee for Electrotechnical Standarization, 2012. 